\title{
Gastrointestinal Hemorrhage in Patients with Acute Ischemic Stroke: Should Endoscopy Be Within the Scope of Practice?
}

\author{
Paul Leonor $^{1} \cdot$ Ara B. Sahakian ${ }^{1}$ \\ Published online: 7 February 2019 \\ (c) Springer Science+Business Media, LLC, part of Springer Nature 2019
}

Approximately 795,000 strokes occur in the USA each year, $87 \%$ of which are ischemic [1]. The management of acute ischemic stroke (AIS) may involve thrombolytic agents in patients identified as appropriate candidates [2]. Many patients are subsequently placed on secondary prophylaxis with antiplatelet agents. Anticoagulation with warfarin or novel oral anticoagulants may be used in cases of atrial fibrillation [3, 4]. As such, these patients are at a small, albeit clinically significant increased risk of gastrointestinal hemorrhage (GIH). GIH in the setting of AIS is associated with higher in-hospital mortality and increased odds of severe long-term dependence $[5,6]$. This poses a difficult clinical decision for gastroenterologists. The approach to GIH in AIS is not specifically addressed by gastroenterology societal guidelines and has generally been considered a relative contraindication to gastrointestinal endoscopy (GIE). Unfortunately, there has been a lack of data thus far to help guide endoscopists caring for patients with GIH in the setting of AIS.

Siddiqui et al. [7] attempt to tackle this challenging clinical scenario in their retrospective analysis entitled "Endoscopy is Relatively Safe in Patients with Acute Ischemic Stroke and Gastrointestinal Hemorrhage" in this issue of Digestive Diseases and Sciences. The authors utilized 10 years of data from the Nationwide Inpatient Sample database to evaluate 75,756 hospitalizations of patients with a diagnosis of AIS and concurrent GIH as identified by International Classification of Diseases (ICD)-9 clinical modification (-CM) codes. They subsequently compared outcomes between patients that underwent GIE versus those that did not. The primary outcome measured was in-hospital mortality, and the secondary outcomes were resource utilization,

Ara B. Sahakian

arasahak@med.usc.edu

1 Division of Gastrointestinal and Liver Diseases, University of Southern California School of Medicine, 1510 San Pablo Street, Los Angeles, CA 90033, USA measured by length of stay (LOS) and total costs, and identification of predictors of patients undergoing GIE.

In their analysis, $37.4 \%$ of hospitalized patients with AIS and GIH underwent GIE with a mortality of $9 \%$ compared to $19.5 \%$ for those that did not $(p<0.001)$. In their multivariate regression analysis, the adjusted odds ratio between the groups was $0.4(p<0.001)$ after adjusting for potential confounding variables. They also found that patients who underwent GIE had a statistically significant shorter adjusted LOS of 9.49 versus 10.08 days (mean difference $0.58,95 \%$ CI $0.37-0.80, p<0.001)$ and a lower adjusted cost of hospitalization (mean difference of $\$ 5801, p<0.001$ ). Independent predictors of patients undergoing endoscopy were male gender, age $>65$, Asian or Pacific Islander race, the presence of hypovolemic shock, need for blood transfusion, and admission to urban non-teaching hospitals. Inverse predictors of receiving GIE were the presence of acute respiratory failure, need for mechanical ventilation, and lower severity of illness (Diagnosis-Related Group [DRG] sub-class I to III).

This study provides valuable data and guidance for clinicians that encounter a common clinical dilemma for which evidence in the literature is extremely sparse. Although its greatest strength lies in the robust size of the population studied, it has some limitations that need to be taken into consideration before clinical application of any of its conclusions is recommended. The primary drawbacks of the study lie in its retrospective design and its reliance on ICD9-CM codes. As with any retrospective study utilizing large databases, many confounding factors are present that cannot be accounted for despite valiant efforts directed at their adjustment. As the authors rightfully acknowledged in their discussion, certain individual characteristics that cannot be retrospectively analyzed may have influenced the care team's decision not to pursue GIE while simultaneously contributing to increased mortality. For example, patients that had a more severe stroke, had a poorer prognosis for meaningful neurologic recovery, had poor baseline functional status, or 
who had other life-threatening conditions may have been deemed too unstable to receive GIE. Alternatively, families and providers of these severely ill patients may have opted for less aggressive management given lack of perceived overall benefit. Another potential confounder is whether patients received thrombolysis with tissue plasminogen activator (tPA), as this confers an approximately $6.5 \%$ risk of intracerebral hemorrhage compared to $0.6 \%$ with placebo and a $2 \%$ overall risk of systemic hemorrhage [8]. Patients that develop intracerebral hemorrhage after tPA have as high as $83 \%$ risk of mortality [9] and could conceivably have concurrent GIH but succumb to neurologic deterioration before GIE can be performed.

The analysis of ICD-9-CM codes, although useful, may also introduce potential confounders. Many codes commonly used to define key study variables in large retrospective studies may frequently be invalid. One study that examined administrative database research found that many subjects often have conditions that are miscoded [10]. The converse is also true, as certain conditions may not be coded depending on providers' perception of relevance to the current clinical encounter. This may include or exclude patients in each arm of the study based on the accuracy of coding, or potentially exclude certain comorbid conditions that may have impacted mortality.

This study's greatest strength is perhaps also an important limitation. The vast size of the study's population can inflate seemingly small differences into statistically significant changes. An example of this is readily apparent in the baseline demographics (Table 2 in the article) where the average age of those receiving GIE was 72.2 years versus 74.7 in those that did not $(p<0.001)$. This small difference in age is statistically significant owing to the large sample size. A more clinically relevant example is seen when comparing the LOS between the two groups: The patients that received GIE had a mean of 0.58 days shorter LOS, which was again found to be statistically significant. One could question whether some of these differences may be due to chance, yet appear statistically significant due to the laws of large numbers.

Siddiqui et al. have clarified a topic within the field of gastroenterology in which little evidence is available to support decision-making. Their findings suggest that GIE may be pursued safely in select patients with AIS and GIH with potential improvement in mortality and decreased burden on the health care system. Due to the retrospective design of the study, many confounders still persist; it cannot be assumed that GIE directly contributes to the mortality benefit seen in this study, although the association is encouraging. As aforementioned, due to the large study population, the statistically significant findings must be interpreted with caution as some may be of little clinical impact and confounding factors may exist. Overall, the investigators' findings are promising but need to be further validated with randomized prospective studies.

Author's contribution Leonor was involved in drafting of the article; Sahakian was involved in key editing and revision.

\section{Compliance with ethical standards}

Conflict of interest All authors declare that they have no conflict of interest.

\section{References}

1. Benjamin EJ, Blaha MJ, Chiuve SE, et al. Heart disease and stroke statistics-2017 update: a report from the American Heart Association. Circulation. 2017;135:e146-e603.

2. Powers WJ, Rabinstein AA, Ackerson T, et al. 2018 guidelines for the early management of patients with acute ischemic stroke: a guideline for healthcare professionals from the American Heart Association/American Stroke Association. Stroke. 2018;49:e46-e110.

3. Antithrombotic Trialists C, Baigent C, Blackwell L, et al. Aspirin in the primary and secondary prevention of vascular disease: collaborative meta-analysis of individual participant data from randomised trials. Lancet. 2009;373:1849-1860.

4. January CT, et al. 2014 AHA/ACC/HRS guideline for the management of patients with atrial fibrillation: executive summary: a report of the American College of Cardiology, American Heart Association Task Force on practice guidelines and the Heart Rhythm Society. Circulation. 2014;130:2071-2104.

5. O’Donnell MJ, Kapral MK, Fang J, Saposnik G, et al. Gastrointestinal bleeding after acute ischemic stroke. Neurology. 2008;71:650-655.

6. Rumalla K, Mittal M. Gastrointestinal bleeding in acute ischemic stroke: a population-based analysis of hospitalizations in the United States. J Stroke Cerebrovasc Dis. 2016;25:1728-1735.

7. Siddiqui MT, Bilal M, Gollapudi LA, et al. Endoscopy is relatively safe in patients with acute ischemic stroke and gastrointestinal hemorrhage. Dig Dis Sci. (Epub ahead of print). https://doi. org/10.1007/s10620-018-5399-3.

8. The NINDS t-PA Stroke Study Group. Intracerebral hemorrhage after intravenous t-PA therapy for ischemic stroke. Stroke. 1997;28(11):2109-2118.

9. Kase CS, Furlan AJ, Wechsler LR, et al. Cerebral hemorrhage after intra-arterial thrombolysis for ischemic stroke: the PROACT II trial. Neurology. 2001;57:1603-1610.

10. van Walraven C, Bennett C, Forster AJ. Administrative database research infrequently used validated diagnostic or procedural codes. J Clin Epidemiol. 2011;64:1054.

Publisher's Note Springer Nature remains neutral with regard to jurisdictional claims in published maps and institutional affiliations. 